\title{
Finite Fourier transform for solving potential and steady-state temperature problems
}

Kamel Al-Khaled*

\section{*Correspondence:}

kamel@just.edu.jo

Department of Mathematics and

Statistics, Jordan University of

Science and Technology, Irbid,

Jordan

\begin{abstract}
The derivation of this paper is devoted to describing the operational properties of the finite Fourier transform method, with the purpose of acquiring a sufficient theory to enable us to follow the solutions of boundary value problems of partial differential equations, which has some applications on potential and steady-state temperature. Numerical calculations show that the present method gives higher accuracy with less computation time than other, traditional methods, like the finite difference method.
\end{abstract}

Keywords: Finite Fourier transform; Heat equation; Numerical solutions; Steady-state temperature

\section{Introduction}

Many boundary value problems can be solved by means of integral transformations, such as the Laplace transform function, which transform a differential equation into an algebraic equation in which the boundary conditions are automatically considered. After solving the algebraic equation, one finds the solution of the original equation by means of the inverse transformation. Similarly, partial differential equations are changed into ordinary differential equations by applying these transformations. Two transformations which are particularly useful in solving boundary value problems are the finite Fourier sine and cosine transformations.

The particular transformation discussed in this paper is the finite Fourier transform, which is applicable to equations in which only the even order derivatives (of the function) with respect to transformed variable will be treated. The finite Fourier transform method is one of various analytical techniques in which exact solutions of boundary value problems can be constructed. The transform exists for all bounded, piecewise continuous functions over a finite interval. In recent years, the finite Fourier transform method has been applied to a wide class of boundary value problems in many interesting mathematics, physics, chemistry and engineering areas [1-3]. Many other transforms exist which may be used to solve PDEs $[4,5]$.

A feature which makes the finite transform a very economical method, is that the inverse transform may be solved only for regions of interest [6-8]. The most widely used methods for the solution of boundary value problems are based on finite differences. These 
methods require certain assumptions about where the finite difference equals the derivative which by necessity have to be most loosely made on the boundaries. One must form many grid points near the boundary in order for the numerical solution to be accurate. Another feature of the finite Fourier transform method is that it gives the exact solution at the boundary [9].

This paper is written in two parts. Sect. 2 will be devoted to a description of the operational properties of these transformations with the purpose of acquainting the reader with sufficient theory to enable us to follow the solutions of the problems given in Sect. 3, which consists of a group of simple boundary value problems and their solutions.

\section{Basic definitions}

Definition 1 Let $F(x)$ be a function that is sectionally continuous in the interval $0<x<\pi$, the finite Fourier sine and cosine transformations are defined as follows:

$$
\begin{aligned}
& \mathbf{S}\{F(x)\}=\int_{0}^{\pi} F(x) \sin n x d x=f_{s}(n), \\
& \mathbf{C}\{F(x)\}=\int_{0}^{\pi} F(x) \cos n x d x=f_{c}(n) .
\end{aligned}
$$

The transformations set up a correspondence between functions $F(x)$ on the interval $0<x<\pi$ and the sequences $\left\{f_{s}(n), f_{c}(n)\right\}$ called the finite sine transform and finite cosine transform, respectively, of $F(x)$. From the definition of the transformations it is apparent that they are linear.

\subsection{Some operational properties}

The usefulness of the finite Fourier sine and cosine transformations in solving differential equations is primarily due to the fact that differentiation of $F(x)$ corresponds to a simple algebraic operation on the transform $f_{s}(n)$ or $f_{c}(n)$. If $F(x)$ is continuous and $F^{\prime}(x)$ is sectionally continuous

$$
S\left\{F^{\prime}(x)\right\}=\int_{0}^{\pi} F^{\prime}(x) \sin n x d x=\left.F(x) \sin n x\right|_{n} ^{\pi}-n \int_{0}^{\pi} F(x) \cos n x d x
$$

and hence

$$
S\left\{F^{\prime}(x)\right\}=-n f_{c}(n)
$$

Similarly,

$$
C\left\{F^{\prime}(x)\right\}=-n f_{s}(n)+(-1)^{n} F(\pi)-F(0) .
$$

If $F^{\prime}(x)$ is continuous and $F^{\prime \prime}(x)$ is sectionally continuous, we find upon replacing $F(x)$ by $F^{\prime}(x)$ in Eq. (2.3) and making use of Eq. (2.4)

$$
S\left\{F^{\prime \prime}(x)\right\}-=-n^{2} f_{s}(n)+n\left[F(0)-(-1)^{n} F(\pi)\right] .
$$


Similarly, by starting with Eq. (2.4) we have

$$
C\left\{F^{\prime \prime}(x)\right\}=-n^{2} f_{c}(n)-F^{\prime}(0)+(-1)^{n} F^{\prime}(\pi) .
$$

If $F^{\prime \prime \prime}(x)$ is continuous and $F^{\prime \prime \prime \prime}(x)$ is sectionally continuous, we may replace $F(x)$ by $F^{\prime \prime}(x)$ in (2.5) to obtain

$$
S\left\{F^{\prime \prime \prime \prime}(x)\right\}=n^{4} f_{s}(n)-n^{3}\left[F^{\prime \prime}(0)-(-)^{n} F(\pi)\right]+\left[F^{\prime \prime}(0)-(-1)^{n} F^{\prime \prime}(\pi)\right] .
$$

In like manner, we can find the sine transform of any even-ordered derivatives of $F(x)$ in terms of the sine transform of $F(x)$ and the value of the even-ordered derivatives at the endpoints of the interval. By repeated applications of (2.6) we are able to express the cosine transform of any even-ordered derivative of $F(x)$ in terms of $f_{c}(n)$ at the endpoints of the interval. Another useful property of the Fourier transform will now be developed. Let $F(x)$ be defined in the interval $0<x<\pi$. Let $G(x)$ be the even periodic extension of $F(x)$, then

$$
f_{c}(n) \cos n k=\frac{1}{4} \int_{-\pi}^{\pi} G(x)[\cos n(x+k)+\cos n(x-k) \cos n k] d x .
$$

Breaking the integrand into two integrals, letting $y=s+k$ in the first and $y=x-k$ in the second, this can be written as

$$
\begin{aligned}
& \frac{1}{4} \int_{-\pi+k}^{\pi+k} G(y-k) \cos n y d y+\frac{1}{4} \int_{-\pi-k}^{\pi-k} G(y+k) \cos n y d y \\
& \quad=\frac{1}{4} \int_{-\pi}^{\pi} G(y-k) \cos n y d y+\frac{1}{4} \int_{-\pi}^{\pi} G(y+k) \cos n y d y,
\end{aligned}
$$

because of the fact that the integrand is periodic with period $2 \pi$. Hence $f_{c}(n) \cos n k=$ $I_{1}+I_{2}+I_{3}+I_{4}$ where

$$
\begin{array}{rlrl}
I_{1} & =\frac{1}{4} \int_{-\pi}^{0} G(y-k) \cos n y d y, & I_{2} & =\frac{1}{4} \int_{0}^{\pi} G(y-k) \cos n y d y, \\
I_{3} & =\frac{1}{4} \int_{-\pi}^{0} G(y+k) \cos n y d y, & I_{4}=\frac{1}{4} \int_{0}^{\pi} G(y+k) \cos n y d y .
\end{array}
$$

Substituting $y=-x$ in $I_{1}$ and $I_{3}$ we have

$$
\begin{aligned}
& I_{1}=\frac{1}{4} \int_{\pi}^{0} G(-x-k) \cos n(-x)(-d x)=\frac{1}{4} \int_{0}^{\pi} G(x+k) \cos n x d x=I_{4}, \\
& I_{3}=\frac{1}{4} \int_{\pi}^{0} G(-x+k) \cos n(-x)(-d x)=\frac{1}{4} \int_{0}^{\pi} G(x-k) \cos n x d x=I_{2} .
\end{aligned}
$$

Therefore,

$$
f_{c}(n) \cos n k=C\left\{\frac{1}{2}[G(x+k)+G(x-k)]\right\} .
$$


Let $k=\pi$, we get $(-1)^{n} f_{c}(n)=C\left\{\frac{1}{2}[G(x+\pi)+G(x-\pi)]\right\}$, but $G(x+\pi)=G(x-\pi)$ for all $x$, and $G(\pi-x)=F(\pi-x)$ for all $0<x<\pi$. Therefore

$$
(-1)^{n} f_{c}(n)=C\{F(\pi-x)\}
$$

In a similar manner it can be shown that if $H(x)$ is the odd periodic extension of $F(x)$ of period $2 \pi$; then

$$
(-1)^{n+1} f_{s}(n)=S\{F(\pi-x)\}
$$

\subsection{Convolution}

It is necessary to find the inverse function of the product of two transforms whose inverses are known. To solve this problem, we define the convolution of a function. Let $P(x)$ be defined on the interval $-2 \pi<x<2 \pi$ and let $Q(x)$ be defined on the interval $-\pi<x<\pi$. Then the function

$$
\phi(x)=\int_{-\pi}^{\pi} P(x-\xi) Q(\xi) d \xi \equiv P(x) * Q(x)
$$

is called "the convolution" of $P$ and $Q$ on the interval $-\pi<x<\pi$. It can easily be shown that $\phi(x)$ is an even function if $P$ and $Q$ are both odd or both even, and is odd if one of these functions is even and the other odd. The importance of convolution is brought out in the following theorem.

Theorem 2.1 Let $F(x)$ and $G(x)$ be two functions sectionally continuous on the interval $0 \leq x \leq \pi$. Let $F_{1}(x)$ and $F_{2}(x)$ denote the odd and even periodic extensions of $F$, respectively, and $G_{1}(x)$ and $G_{2}(x)$ the odd and even extensions of $G$. Then the products of the transformations of $F$ and $G$ can be written as follows:

$$
\begin{aligned}
& f_{s}(n) g_{c}(n)=S\left\{\frac{1}{2} F_{1} * G_{2}\right\}, \\
& f_{s}(n) g_{s}(n)=C\left\{\frac{1}{2} F_{1} * G_{1}\right\}, \\
& f_{c}(n) g_{c}(n)=C\left\{\frac{1}{2} F_{2} * G_{2}\right\}, \\
& f_{c}(n) g_{s}(n)=S\left\{\frac{1}{2} F_{2} * G_{1}\right\} .
\end{aligned}
$$

Furthermore, if

$$
\begin{array}{ll}
I_{1}=\int_{0}^{\pi} F(x-\xi) G(\xi) d \xi, & I_{2}=\int_{x}^{\pi} F(\xi-x) G(\xi) d \xi \\
I_{3}=\int_{0}^{\pi-x} F(x+\xi) G(\xi) d \xi, & I_{4}=\int_{\pi-x}^{\pi} F(2 \pi-x-\xi) G(\xi) d \xi
\end{array}
$$

then

$$
F_{1} * G_{2}=I_{1}-I_{2}+I_{3}-I_{4},
$$


Figure 1 Region of integration for Theorem 2.1

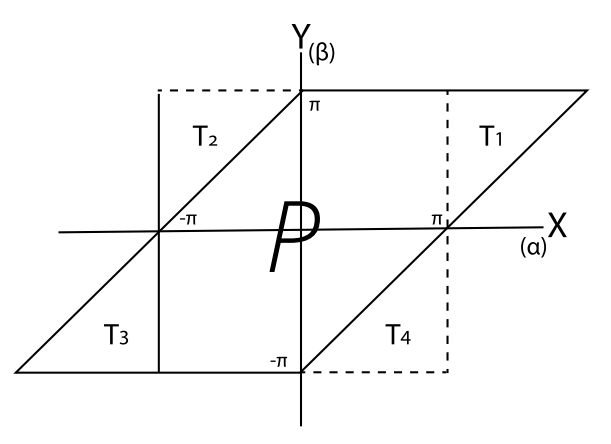

$$
\begin{aligned}
& F_{1} * G_{1}=I_{1}-I_{2}-I_{3}+I_{4}, \\
& F_{2} * G_{2}=I_{1}+I_{2}+I_{3}+I_{4}, \\
& F_{2} * G_{1}=I_{1}+I_{2}-I_{3}+I_{4} .
\end{aligned}
$$

Proof The proof of the theorem will only be illustrated by proving Eqs. (2.15) and (2.19). We have

$$
\begin{aligned}
f_{c}(n) g_{s}(n) & =\int_{0}^{\pi} F_{2}(\alpha) \cos n \alpha d \alpha \int_{0}^{\pi} G_{1}(\beta) \sin n \beta d \beta \\
& =\frac{1}{4} \int_{-\pi}^{\pi} F_{2}(\alpha) \cos n \alpha d \alpha \int_{-\pi}^{\pi} G_{1}(\beta) \sin n \beta d \beta \\
& =\frac{1}{4} \int_{-\pi}^{\pi} \int_{-\pi}^{\pi} F_{2}(\alpha) G_{1}(\beta) \cos n \alpha \sin n \beta d \alpha d \beta .
\end{aligned}
$$

If we let $S$ represent the square bounded by the lines $\alpha=\mp \pi, \beta=\mp \pi$ (see Fig. 1), we have

$$
\begin{aligned}
f_{c}(n) g_{s}(n)= & \frac{1}{8} \iint_{S} F_{2}(\alpha) G_{1}(\beta) \sin n(\alpha+\beta) d \alpha d \beta \\
& -\frac{1}{8} \iint_{S} F_{2}(\alpha) G_{1}(\beta) \sin n(\alpha-\beta) d \alpha d \beta
\end{aligned}
$$

In the first integrand let $\alpha+\beta=x$ and $\beta=y$, in the second integrand let $\alpha-\beta=x$ and $\beta=-y$. The Jacobian of the first transformation is 1 ; that of the second is -1 . So we have

$$
\begin{aligned}
f_{c}(n) g_{s}(n)= & \frac{1}{8} \iint_{P} F_{2}(x-y) G_{1}(y) \sin n x d x d y \\
& +\frac{1}{8} \iint_{P} F_{2}(x-y) G_{1}(-y) \sin n x d x d y .
\end{aligned}
$$

For any fixed $y(-\pi \leq y \leq \pi)$, the integrands are periodic functions of $x$ with period $2 \pi$, hence the integral over $T_{1}$ can be replaced by the integral over $T_{2}$ and the integral over $T_{3}$ can be replaced by the integral over $T_{4}$. The resulting integral can be written as

$$
\begin{aligned}
& \frac{1}{8} \int_{-\pi}^{\pi} \sin n x \int_{-\pi}^{\pi} F_{2}(x-y) G_{1}(y) d y d x-\frac{1}{8} \int_{-\pi}^{\pi} \sin n x \int_{\pi}^{-\pi} F_{2}(x-y) G_{1}(y) d y d x \\
& \quad=\frac{1}{4} \int_{-\pi}^{\pi} \sin n x \int_{-\pi}^{\pi} F_{2}(x-y) G_{1}(y) d y d x
\end{aligned}
$$




$$
\begin{aligned}
& =\frac{1}{2} \int_{0}^{\pi} \sin n x \int_{-\pi}^{\pi} F_{2}(x-y) G_{1}(y) d y d x \\
& =\frac{1}{2} S\left\{F_{2} * G_{1}\right\}=S\left\{\frac{1}{2} F_{2} * G_{1}\right\},
\end{aligned}
$$

where we have used the fact that $F_{2} * G_{1}$ is an odd function. We have

$$
\begin{aligned}
F_{2} * G_{1}= & \int_{0}^{x} F_{2}(x-\xi) G_{1}(\xi) d \xi+\int_{x}^{\pi} F_{2}(x-\xi) G_{1}(\xi) d \xi \\
& +\int_{-\pi+x}^{0} F_{2}(x-\xi) G_{1}(\xi) d \xi+\int_{-\pi}^{-\pi+x} F_{2}(x-\xi) G_{1}(\xi) d \xi
\end{aligned}
$$

In the third and fourth integrals replace $\xi$ by $-\xi$,

$$
\begin{aligned}
F_{2} * G_{1}= & \int_{0}^{x} F_{2}(x-\xi) G_{1}(\xi) d \xi+\int_{x}^{\pi} F_{2}(\xi-x) G_{1}(\xi) d \xi \\
& +\int_{0}^{\pi-x} F_{2}(x+\xi) G_{1}(-\xi) d \xi+\int_{\pi-x}^{\pi} F_{2}(x+\xi) G_{1}(-\xi) d \xi
\end{aligned}
$$

whence by making use of the definitions of the extended functions $F_{2}$ and $G_{1}$, Eq. (2.19) follows immediately.

\section{Solutions of partial differential equations}

Since we will be concerned with functions of two independent variables, it is necessary to comment briefly on the characteristic of the finite Fourier transform of such a function. The finite Fourier sine transformation of $F(x, t),(0<x<\pi)$ is

$$
S\{F(x, t)\} \equiv \int_{0}^{\pi} F(x, t) \sin n x d x \equiv f(n, t) \quad(n=1,2,3, \ldots) .
$$

If $\frac{\partial^{n}}{\partial t^{n}} F(x, t)$ exists and is continuous then

$$
S\left\{\frac{\partial^{n} F(x, t)}{\partial t^{n}}\right\}=\int_{0}^{\pi} \frac{\partial^{n}}{\partial t^{n}} F(x, t) \sin n x d x=\frac{d^{n}}{d t^{n}}\left\{\int_{0}^{\pi} F(x, t) \sin n x d x\right\} \equiv \frac{d^{n}}{d t^{n}} f(n, t) .
$$

All the formulas listed in the tables made for single functions [10] can be extended very easily to the case where $F$ is a function of two independent variables. For example

$$
S\left\{\frac{\partial^{2} F(x, t)}{\partial x^{2}}\right\}=-n^{2} f(n, t)+n\left[F(0, t)-(-1)^{n} F(\pi, t)\right] .
$$

Similar changes must be made in the other formulas in the applications which follow. In all these applications the sine transform, rather than the cosine transform, is used since the boundary conditions include the value of the function at the endpoints of the interval.

\subsection{Vibrations of a horizontal string with fixed ends}

A constant transverse force acts at each point of a string with ends $x=0$ and $x=\pi$ fixed; thus we need to solve the problem

$$
Y_{t t}=a^{2} Y_{x x}+F(x)
$$


subject to the conditions $Y(0, t)=Y(\pi, t)=0$, and $Y(x, 0)=Y_{t}(x, 0)=0$.

Applying the sine transformation to Eq. (3.1) and using the boundary conditions yields the differential equation

$$
\frac{d^{2}}{d t^{2}} y(n, t)=-n^{2} a^{2} y(n, t)+f(n),
$$

which has the solution

$$
y(n, t)=C_{1} \sin n a t+C_{2} \cos n a t+\frac{f(n)}{a^{2} n^{2}} .
$$

In order to satisfy the initial conditions, we choose $C_{1}=0$ and $C_{2}=\frac{-f(n)}{a^{2} n^{2}}$. Define $G(x)$ such that $G^{\prime \prime}(x)=F(x),(0<x<\pi)$ and $G(0)=G(\pi)=0$, we obtain $\frac{f(n)}{n^{2}}=\frac{S\left\{G^{\prime \prime}(x)\right\}}{n^{2}}=-g(n)$. Therefore

$$
y(n, t)=\frac{g(n)}{a^{2}} \cos n a t-\frac{g(n)}{a^{2}} .
$$

Hence

$$
Y(x, t)=\frac{-1}{2 a^{2}}[G(x-a t)+G(x+a t)]-\frac{G(x)}{a^{2}}
$$

where $G(-x)=-G(x)$ and $G(x+2 \pi)=G(x)$.

\subsection{Transverse vibrations of a beam}

If a constant transverse force acts at each point of a beam, the transverse displacements $Y(x, t)$ satisfy the equation

$$
\frac{\partial^{2} Y}{\partial t^{2}}=-a^{2} \frac{\partial^{2} Y}{\partial x^{2}}+F(x) .
$$

If the ends, $x=0$ and $x=\pi$, are hinged so that $Y$ and $Y_{x x}$ vanish there, and if the initial displacement and velocity are zero, we may find $Y(x, t)$ by applying the sine transformations to Eq. (3.2) and considering the boundary conditions

$$
Y(0, t)=Y(\pi, t)=0, \quad Y_{x x}(0, t)=Y_{x x}(\pi, t)=0 .
$$

Therefore, we obtain

$$
\frac{d^{n}}{d t^{2}} y(n, t)=-a^{2} n^{2} y(n, t)+f(n) .
$$

Hence $y(n, t)=a n^{2} C_{1} \cos a n^{2} t-a n^{2} C_{2} \sin a n^{2} t$. To satisfy the conditions $Y(x, 0)=Y_{t}(x, 0)=$ 0 , we choose $C_{1}=0 ; C_{2}=-\frac{f(n)}{a^{2} n^{2}}$. Therefore

$$
y(n, t)=\frac{f(n)}{a^{2} n^{2}}\left(1-\cos a n^{2} t\right) .
$$


If we define $G(x)$ such that $G^{\prime \prime \prime \prime}(x)=F(x), 0<x<\pi ; G(x)=G^{\prime \prime}(x)=0$ at $x=0$ and $x=\pi$. Then $\frac{f(n)}{n^{2}}=g(n)$, and so $y(n, t)=\frac{g(n)}{a^{2}}-\frac{f(n)}{a^{2} n^{2}} \cos a n^{2} t$. Therefore our solution is given as

$$
Y(x, t)=\frac{G(x)}{a^{2}}-\frac{2}{\pi a^{2}} \sum_{n=1}^{\infty} \frac{f(n)}{n^{4}} \cos a n^{2} t \sin n x .
$$

\subsection{Applications to problems in heat conduction}

Application 1 As an application, we would like to find the temperature distribution $U(x, t)$ of a bar 30 centimeters in length after the ends are placed in contact with ice if the sides are insulated and the initial temperature distribution is defined as follows:

$$
U(x, 0)= \begin{cases}0^{\circ}, & 0<x<10 ; 20<x<30 \\ 100^{\circ}, & 10<x<20\end{cases}
$$

The differential equation which $U(x, t)$ must satisfy is

$$
U_{t}(x, t)=a^{2} U_{x x}(x, t)
$$

If we choose a new unit of length such that $1=30 / \pi \mathrm{cm}$, the rod becomes $\pi$ units long and the boundary conditions become

$$
\begin{aligned}
& U(0, t)=U(\pi, t)=0, \quad t>0, \\
& U(x, 0)= \begin{cases}0^{\circ}, & 0<x<\frac{\pi}{3}, \frac{2 \pi}{3}<x<\pi, \\
100^{\circ}, & \frac{\pi}{3}<x<\frac{2 \pi}{3} .\end{cases}
\end{aligned}
$$

Applying the sine transformation to Eqs. (3.3) and (3.4) we obtain $\frac{d}{d t} u(n, t)=-a^{2} n^{2} u(n, t)$, which has the solution $u(n, t)=C \exp \left(-a^{2} n^{2} t\right)$. To determine the constant $C$, we make use of the condition in (3.5), as

$$
C=u(n, 0)=\int_{0}^{\pi} U(x, 0) \sin n x d x=\int_{\pi / 3}^{2 \pi / 3} 100 \sin n x d x=\frac{100}{n}\left(2 \sin \frac{n \pi}{2} \sin \frac{n \pi}{6}\right) .
$$

For $n=2 k$, we have $\sin \frac{2 k \pi}{2}=0$, therefore $C_{2 k}=0$. While, for $n=2 k+1, \sin \frac{(2 k+1) \pi}{2}=(-1)^{k}$, which implies that $C_{2 k+1}=\frac{200(-1)^{k} \sin \frac{(2 k+1) \pi}{2}}{2 k+1}$. We arrive at

$$
u(2 k+1, t)=\frac{200(-1)^{k} \sin \frac{(2 k+1) \pi}{2}}{2 k+1} \exp \left[-a^{2}(2 k+1)^{2} t\right] .
$$

The temperature distribution $U(x, t)$ of the rod is given by

$$
U(x, t)=\frac{400}{\pi} \sum_{k=0}^{\infty}(-1)^{k} \frac{\sin \frac{(2 k+1) \pi}{6}}{2 k+1} \exp \left[-a^{2}(2 k+1)^{2} t\right] \sin (2 k+1) x .
$$

The solution of this problem for a bar for which $a^{2}=0.185$ and $a^{2}=0.1$ (c.g.s. system) is displayed in Fig. 2. Since the solution is symmetrical about the line $x=\pi / 2(15 \mathrm{~cm})$ the 

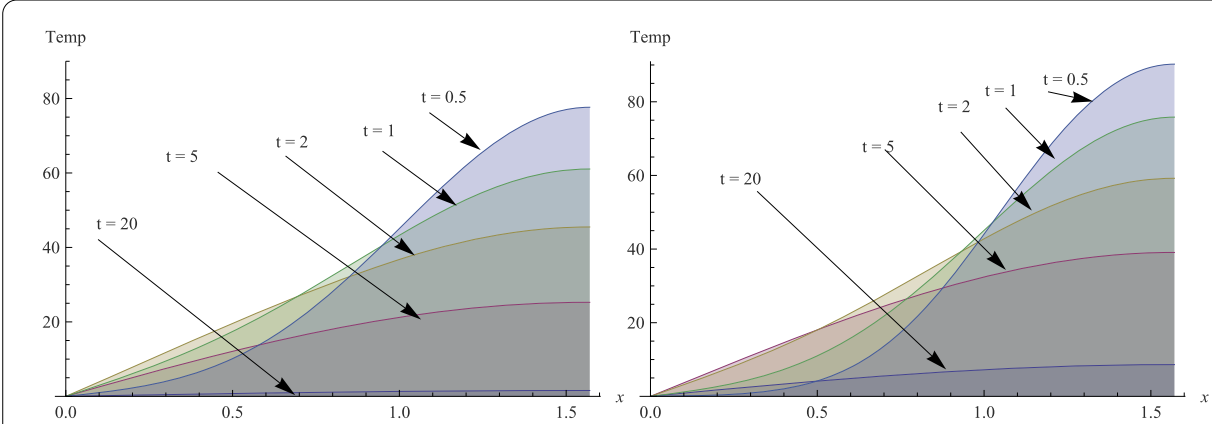

Figure 2 Right graph represent the temperature when $t=20,5,2,1,0.5$ minutes for $a^{2}=0.185$, while the left one is for $a^{2}=0.10$ for the same values of $t$

graph extends over only one-half the bar. The constant $a^{2}$ must be based upon the same unit of measurement as $x$. If its value is known in the c.g.s. system, it must be multiplied by $\pi^{2} / 900$ before being used in this formula.

Application 2 We seek the temperature $U(x, t)$ which satisfies the conditions

$$
U_{t}(x, t)=U_{x x}(x, t)-h(t) U(x, t)+A, \quad 0<x<\pi, t>0,
$$

subject to the boundary conditions

$$
U(0, t)=U(\pi, t)=0
$$

and the initial condition

$$
U(x, 0)=0 .
$$

By means of the sine transformation, the above problem becomes

$$
\frac{d}{d t} u(n, t)=-n^{2} u(n, t)-h(t) u(n, t)+A S\{1\}, \quad u(n, 0)=0 .
$$

Hence,

$$
\frac{d}{d t} u(n, t)+\left\{n^{2}+h(t)\right\} u(n, t)=A \frac{1-(-1)^{n}}{n} .
$$

This is an ordinary linear differential equation whose solution is

$$
u(n, t)=A \frac{1-(-1)^{n}}{n} \exp \left[-\int\left\{n^{2}+h(t)\right\} d t\right] \int \exp \left[\int\left\{n^{2}+h(t)\right\} d t\right] d t
$$

Since $t \geq 0$, and we wish $u(n, 0)$ to be zero, we may write the answer in the following form:

$$
u(n, t)=A \exp \left[-\int_{0}^{t} h(\tau) d \tau\right] \int_{0}^{t} \exp \left[\int_{0}^{\tau} h(\tau) d \tau\right] \frac{1-(-1)^{n}}{n} \exp \left[-n^{2}(t-\tau)\right] d \tau
$$


If we let $H(t)=\int_{0}^{t} h(\tau) d \tau$, and $\Psi(x, t)=\frac{2}{\pi} \sum_{n=1}^{\infty} \frac{1-(-1)^{n}}{n} \exp \left[-n^{2} t\right] \sin n x$, then

$$
U(x, t)=A \exp [-H(t)] \int_{0}^{t} \exp [H(\tau)] \Psi(x, t-\tau) d \tau
$$

Application 3 Let $\theta(x, t)$ be the temperature function that satisfies

$$
\theta_{t}(x, t)=\theta_{x x}(x, t), \quad 0<x<\pi, t>0,
$$

subject to the boundary and initial conditions

$$
\theta(0, t)=\theta(\pi, t)=0, \quad \theta(x, 0)=\frac{\pi-x}{\pi} .
$$

Application of the sine transformation yields $\frac{d}{d t} \theta(n, t)=-n^{2} \theta(n, t), \theta(n, 0)=\frac{1}{n}$. The solution of the above differential equation is $\theta(n, t)=\frac{1}{n} \exp \left[-n^{2} t\right]$. Hence $\theta(x, t)=\frac{2}{\pi} \sum_{n=1}^{\infty} \frac{1}{n} \times$ $\exp \left[-n^{2} t\right] \sin n x$.

Let $U(x, t)$ be a solution of the problem

$$
U_{t}(x, t)=U_{x x}(x, t), \quad 0<x<\pi, t>0,
$$

subject to the boundary and initial conditions

$$
U(0, t)=U(\pi, t)=0, \quad U(x, 0)=F(x) .
$$

We wish to obtain the function $U(x, t)$ in terms of $\theta(x, t)$. The value of the sine transform of $U(x, t)$ is found by replacing $\frac{1}{n}$ by $f(n)$ in the expression for $\theta(x, t)$, to obtain

$$
u(n, t)=f(n) \exp \left[-n^{2} t\right]=n f(n) \theta(n, t)
$$

If $F(x)$ is continuous and $F(0)=F(\pi)=0$, then $n f(n)=C\left\{F^{\prime}(x)\right\}=\int_{0}^{\pi} F^{\prime}(\lambda) \cos n \lambda d \lambda$. Hence, $u(n, t)=\int_{0}^{\pi} F^{\prime}(\lambda) \theta(n, t) \cos n \lambda d \lambda$. Since $\theta(x, t)$ is an odd extension function with period $2 \pi$, we may rewrite $u(n, t)$ as

$$
\begin{aligned}
u(n, t) & =\frac{1}{2} \int_{0}^{\pi} F^{\prime}(\lambda) \int_{0}^{\pi}\{\theta(x+\lambda, t)+\theta(x-\lambda, t)\} \sin n x d x d \lambda \\
& =\frac{1}{2} \int_{0}^{\pi}\left\{\int_{0}^{\pi} F^{\prime}(\lambda)[\theta(x+\lambda, t)+\theta(x-\lambda, t)] d \lambda\right\} \sin n x d x .
\end{aligned}
$$

Therefore $U(x, t)=\frac{1}{2} \int_{0}^{\pi} F^{\prime}(\lambda)[\theta(x+\lambda, t)+\theta(x-\lambda, t)] d \lambda$.

Application 4 The preceding application was generalized by Brown [11]. Consider a cylindrical bar of length $\pi$ which has a diameter so small that the variation of the temperature $F(x, t)$ over every cross section can be neglected. The thermal conductivity of the bar is a function of the time, there is a continuous source of heat along the bar, and the initial temperature at each point of the bar is given by a prescribed function. The rate of loss of heat through the surface at each point is proportional to the temperature at that point. 
The temperature at the ends, $x=0$ and $x=\pi$ are determined by prescribed functions of $t$. The boundary value problem for the temperature function $F(x, t)$ can be written as

$$
\Phi(F) \equiv \frac{\partial F}{\partial t}-C_{1}(t) \frac{\partial^{2} F}{\partial x^{2}}+C_{2}(t) F=P(x, t), \quad 0<x<\pi, t>0,
$$

Subject to

$$
F(0, t)=C_{3}(t), \quad F(\pi, t)=C_{4}(t), \quad t>0, \quad F(x, 0)=G(x), \quad 0<x<\pi .
$$

The solution of the above boundary value problem can be written as

$$
F(x, t)=F_{1}(x, t)+F_{2}(x, t)+F_{3}(x, t)+F_{4}(x, t)
$$

where $F_{1}, F_{2}, F_{3}$ and $F_{4}$ are solutions of the problems:

$$
\begin{array}{lrrr}
\Phi\left(F_{1}\right)=P(x, t), & F_{1}(0, t)=F_{1}(\pi, t)=F_{1}(x, 0)=0, & \\
\Phi\left(F_{2}\right)=0, & F_{2}(\pi, t)=0, \quad F_{2}(0, t)=C_{3}(t), & F_{2}(x, 0)=0, \\
\Phi\left(F_{3}\right)=0, & F_{3}(\pi, t)=C_{4}(t), & F_{3}(0, t)=0, & F_{3}(x, 0)=0, \\
\Phi\left(F_{4}\right)=0, & F_{4}(\pi, t)=0, & F_{4}(0, t)=0, & F_{2}(x, 0)=G(x) .
\end{array}
$$

Problem (3.12) need not be considered as a separate problem, since its solution can be obtained by replacing $x$ by $\pi-x$ and $C_{3}(t)$ by $C_{4}(t)$ in the solution of problem (3.11). The finite sine transformation applied to the equations of problems (3.10), (3.11) and (3.13) yields the differential equations

$$
\begin{aligned}
& \frac{d}{d t} f_{1}(n, t)+\left[n^{2} C_{1}(t)+C_{2}(t)\right] f_{1}(n, t)=p(n, t), \quad f_{1}(n, 0)=0, \\
& \frac{d}{d t} f_{2}(n, t)+\left[n^{2} C_{1}(t)+C_{2}(t)\right] f_{2}(n, t)-n C_{1}(t) C_{3}(t)=0, \quad f_{2}(n, 0)=0,
\end{aligned}
$$

and

$$
\frac{d}{d t} f_{4}(n, t)+\left[n^{2} C_{1}(t)+C_{2}(t)\right] f_{4}(n, t)=0, \quad f_{4}(n, 0)=g(n) .
$$

Since problem (3.14) and (3.15) are ordinary linear differential equations, their solutions can be found formally. The solution of problem (3.14) is

$$
f_{1}(n, t)=\exp \left[-\int\left\{n^{2} C_{1}(t)+C_{2}(t)\right\} d t\right]\left(\int \exp \left[\int\left\{n^{2} C_{1}(t)+C_{2}(t)\right\} d t\right]\right) p(n, t) d t .
$$

Since $t \geq 0$ and we wish $f_{1}(n, 0)=0$, this may be written for $0 \leq \tau \leq t$ as follows:

$$
\begin{aligned}
f_{1}(n, t)= & \exp \left[-n^{2} \int_{0}^{t} C_{1} d \lambda\right] \exp \left[-\int_{0}^{t} C_{2}(\lambda) d \lambda\right] \\
& \times \int_{0}^{t} \exp \left[n^{2} \int_{0}^{\tau} C_{1}(\lambda) d \lambda\right] \exp \left[\int_{0}^{\tau} c_{2}(\lambda) d \lambda\right] p(n, \tau) d \tau
\end{aligned}
$$


or

$$
f_{1}(n, t)=\int_{0}^{t} \exp \left[-n^{2} \int_{\tau}^{t} C_{1}(\lambda) d \lambda\right] \exp \left[-\int_{\tau}^{t} C_{2}(\lambda) d \lambda\right] p(n, \tau) d \tau
$$

Let $H_{i}(\tau, t)=\int_{\tau}^{t} C_{i}(\lambda) d \lambda$, then

$$
f_{1}(n, t)=\int_{0}^{t} p(n, \tau) \exp \left[-n^{2} H_{1}(\tau, t)\right] \exp \left[-H_{2}(\tau, t)\right] d \tau
$$

By analogy we see that the solution of problem (3.15) is

$$
f_{2}(n, t)=\int_{0}^{t} n C_{1}(\tau) C_{3}(\tau) \exp \left[-n^{2} H_{1}(\tau, t)\right] \exp \left[-H_{2}(\tau, t)\right] d \tau .
$$

The solution of the separable differential equation in (3.16) is given by

$$
f_{4}(n, t)=A \exp \left[-n^{2} \int_{0}^{t} C_{1}(\lambda) d \lambda\right] \exp \left[-\int_{0}^{t} C_{2}(\lambda) d \lambda\right] \text {. }
$$

Since $f(n, 0)=g(n), A=g(n)$. Hence the solution is

$$
f_{4}(n, t)=g(n) \exp \left[-n^{2} H_{1}(0, t)\right] \exp \left[-H_{2}(0, t)\right]
$$

By applying the inversion formula to Eqs. (3.17), (3.18) and (3.19), each of the component solutions of $F(x, t)$ is obtained. However, it will be now be shown that the solution of each of the problems (3.10), (3.11) and (3.13) can be expressed in terms of $F_{0}(x, t)$, which is the solution of the simple boundary value problem

$$
\begin{aligned}
& \frac{\partial F_{0}}{\partial t}=\frac{\partial^{2} F_{0}}{\partial x^{2}}, \quad 0<x<\pi, t>0, \\
& F_{0}(0, t)=F_{0}(\pi, t)=0, \quad t>0, \quad F_{0}(x, 0)=\frac{\pi-x}{\pi}
\end{aligned}
$$

whose solution as seen from Application 3 is $f_{0}(n, t)=\frac{1}{n} \exp \left[-n^{2} t\right]$. From this equation we obtain the identities

$$
\exp \left[-n^{2} H_{i}(\tau, t)\right]=n f_{0}\left[n, H_{i}(\tau, t)\right], \quad \exp \left[-n^{2} H_{i}(0, t)\right]=n f_{0}\left[n, H_{i}(0, t)\right]
$$

Differentiating the first equation with respect to $\tau$ we obtain

$$
-n^{2} \exp \left[-n^{2} H_{i}(\tau, t)\right]\left[-C_{i}(\tau)\right]=n \frac{\partial}{\partial \tau} f_{0}\left[n, H_{i}(\tau, t)\right]
$$

or

$$
n C_{i}(\tau) \exp \left[-n^{2} H_{i}(\tau, t)\right]=\frac{\partial}{\partial \tau} f_{0}\left[n, H_{i}(\tau, t)\right]
$$

Therefore, we can express $f_{1}(n, t), f_{2}(n, t)$ and $f_{4}(n, t)$ as functions of $f_{0}(n, t)$ as follows:

$$
f_{1}(n, t)=\int_{0}^{t} \exp \left[-H_{2}(\tau, t)\right] n f_{0}\left[n, H_{1}(\tau, t)\right] p(n, \tau) d \tau
$$




$$
\begin{aligned}
& f_{2}(n, t)=\int_{0}^{t} C_{3}(\tau) \exp \left[-H_{2}(\tau, t)\right] \frac{\partial}{\partial \tau} f_{0}\left[n, H_{1}(\tau, t)\right] d \tau, \\
& f_{4}(n, t)=\exp \left[-H_{2}(0, t)\right] g(n) n f_{0}\left[n, H_{1}(0, t)\right] .
\end{aligned}
$$

We observe that $n f_{0}\left[n, H_{1}(\tau, t)\right]$ is the cosine transform of $\frac{\partial}{\partial \xi} F_{0}\left[\xi, H_{1}(\tau, t)\right]$ since $F_{0}(0, t)=$ $F_{0}(\pi, t)=0$. If, for values of $x$ outside the interval $(0, \pi)$, we define $\frac{\partial F_{0}}{\partial \xi}$ as the even periodic extension and $P(x, t)$ and $G(x)$ as the odd periodic extensions of the original period $2 \pi$ functions

$$
\begin{aligned}
& F_{1}(x, t)=\frac{1}{2} \int_{0}^{t} \exp \left[-H_{2}(\tau, t)\right] \int_{-\pi}^{\pi} P(x-\xi, \tau) \frac{\partial}{\partial \xi} F_{0}\left[\xi, H_{1}(\tau, t)\right] d \xi d \tau, \\
& F_{4}(x, t)=\frac{1}{2} \exp \left[-H_{2}(0, t)\right] \int_{-\pi}^{\pi} G(x-\xi, \tau) \frac{\partial}{\partial \xi} F_{0}\left[\xi, H_{1}(0, t)\right] d \xi \\
& F_{2}(x, t)=\int_{0}^{t} C_{3}(\tau) \exp \left[-H_{2}(\tau, t)\right] \frac{\partial}{\partial \tau} F_{0}\left[x, H_{1}(\tau, t)\right] d \tau .
\end{aligned}
$$

The solution $F_{3}$ of problem (3.12) can be written

$$
F_{3}(x, t)=\int_{0}^{t} C_{4}(\tau) \exp \left[-H_{2}(\tau, t)\right] \frac{\partial}{\partial \tau} F_{0}\left[\pi-x, H_{1}(\tau, t)\right] d \tau
$$

Hence the solution of the general temperature problem (Application 4) can be resolved into the solution of the simple temperature problem (Application 3) by the formula

$$
F(x, t)=F_{1}(x, t)+F_{2}(x, t)+F_{3}(x, t)+F_{4}(x, t)
$$

where $F_{1}, F_{2}, F_{3}$ and $F_{4}$ are defined above. For verification of the resolution see [11].

\section{Concluding remarks}

Boundary value problems of partial differential equations concerned with temperature as the unknown may be solved by a finite Fourier transform method. The temperature at points other than the boundary, if they should be needed, can be obtained by summing the Fourier coefficients. For potential problems, the temperature at the boundary should be as accurate as possible. The finite Fourier transform method which gives the exact boundary temperature within the computer accuracy is shown to be an extremely powerful mathematical tool for the analysis of boundary value problems of partial differential equations with applications in physics. Also the finite Fourier transform method differs from the usual Fourier transformation method in that the solutions are obtained without performing the inverse Fourier transforms. In principle, the finite Fourier transform method may be extended to analog simulations of heat equations in three space variables, and it may also be a very efficient technique for the solution of multidimensional heat equations.

Acknowledgements

The author is grateful to the editor and the reviewers for their careful reading and useful comments. This research was partially supported by Jordan University of Science and Technology. 
Authors' contributions

The author wrote this paper, read and approved the final manuscript.

\section{Publisher's Note}

Springer Nature remains neutral with regard to jurisdictional claims in published maps and institutional affiliations.

Received: 10 January 2018 Accepted: 9 March 2018 Published online: 20 March 2018

\section{References}

1. Maddah, H.A.: Application of finite Fourier transform and similarity approach in a binary system of the diffusion of water in a polymer. J. Mater. Sci. Chem. Eng. 4, 20-30 (2016)

2. Kobayashi, K., Ohtani, N., Jung, J.: Solution of two-dimensional diffusion equation by the finite Fourier transformation. J. Nucl. Sci. Eng. 55(3), 320-328 (1974) Published online: 13 May 2017

3. Tufa Negero, N.: Fourier transform methods for partial differential equations. Int. J. Partial Differ. Equ. 2(3), 44-57 (2014)

4. Leonard, C.J.: Integral transforms for applications to partial differential equations. Grumman Aircraft Engineering Corporation, Research Department Report RE-103, September 1958

5. Sneddon, I.N.: Fourier Transforms. McGraw-Hill, New York (1951)

6. Maddah, H.A.: Application of finite Fourier transform and similarity approach in a binary system of the diffusion of water in a polymer. Int. Mater. Sci. Chem. Eng. 4, 20-30 (2016)

7. LeVeque, R., LeVeque, R.: Finite Difference Methods for Ordinary and Partial Differential Equations: Steady-State and Time-Dependent Problems. SIAM, Philadelphia (2007)

8. Butzer, P.L., Nessel, R.J.: 7 Fourier transform methods and second-order partial differential equations. Pure Appl. Math. 40, 278-302 (1971)

9. Liban, E.: The application of finite Fourier transforms to analog computer simulation. In: Proceedings of the May 1-3 1962 Spring Joint Computer Conference-AIEE-IRE'62 (Spring), pp. 255-265 ACM, New York (1962). https://doi.org/10.1145/1460833.1460862

10. Agarwal, R., Donal, R.: Ordinary and Partial Differential Eqtautions with Special Functions, Fourier Series, and Boundary Value Problems. Springer, New York (2009)

11. Brown, H.K.: Resolution of temperature problems by the use of finite Fourier transformations. Bull. Am. Math. Soc. 50, 376-385 (1944)

\section{Submit your manuscript to a SpringerOpen ${ }^{\odot}$ journal and benefit from:}

- Convenient online submission

- Rigorous peer review

- Open access: articles freely available online

- High visibility within the field

- Retaining the copyright to your article 